Federal Reserve Bank of Minneapolis

Research Department Staff Report 231

May 1997

\title{
Computation of Equilibria in Heterogeneous Agent Models*
}

\author{
José-Víctor Ríos-Rull \\ Federal Reserve Bank of Minneapolis \\ and University of Pennsylvania
}

\begin{abstract}
This paper essentially puts together procedures that are used in the computation of equilibria in models with a very large number of heterogeneous agents. It is not a complete description of all procedures used in the literature. It describes procedures that deal with infinitely lived agent versions of the growth model with and without aggregate uncertainty, overlapping generations models, and dynamic political economy models.
\end{abstract}

*This paper includes most of the material on computing equilibria in models with heterogeneous agents from lectures given at the European University Institute, the University of California at Los Angeles, and the University of Pennsylvania. The comments of many colleagues and students have been very useful. The author thanks the National Science Foundation for grant SBR-9309514. The views expressed herein are those of the author and not necessarily those of the Federal Reserve Bank of Minneapolis or the Federal Reserve System. 


\section{Introduction}

There are many questions in economics for which heterogeneous agent dynamic models have to be used to provide answers. Examples of those questions where the desired answer is quantitative are

1. What changes in the distribution of wealth will occur if the tax system is changed from progressive to proportional?

2. What increases in taxation are needed to maintain the current level of U.S. Social Security benefits under current population patterns?

3. What type of policy changes can be expected from changes in constitutions?

All these questions require models where the households that populate the economy are not identical. With respect to the first question, note that the key property of progressivity of the tax system is that different households face different tax rates. For the second question, the age distribution of the population determines the amounts collected and paid by the administrators of social security. Finally, the determinants of policy should be affected by the relations between different groups of households that do not have the same preferences over policies. In what follows, I describe the models that have been used to address these and related questions, and I pose the models in ways that are susceptible for computation.

I start with models that address the distribution of wealth. For this, I need a theory of wealth differences. There are many candidates for such a theory, the most obvious of which are based on differences in ages with households saving for retirement, preferences with some households being more patient than others, and luck where households hold assets to smooth consumption when individual luck cannot be insured away. I start with the simplest version of a model of wealth differences based on differences in luck.

\section{A Simple Model of the Wealth Distribution}

Imagine a model economy populated with a large number of households (actually, a continuum). The households care about streams of consumption in a standard way:

$$
E_{0}\left\{\sum_{t=0}^{\infty} \beta^{t} u\left(c_{t}\right)\right\}
$$


where for each period $t, c_{t}$ is restricted to belonging to $C$, the per period consumption possibility set of the agent, which might specify nonnegative consumptions. Every period, households receive an endowment of the consumption good, I'll call it coconuts, $s \in S=\left\{s_{1}, \cdots, s_{n}, \cdots, s_{N}\right\}$ that follows a Markov process with transition matrix $\Gamma$. Note that $s_{t}$ is then a random variable that is independently distributed across individuals. The fact that there are a large number of households guarantees that a law of large numbers holds [see Uhlig (1996)] and that the fraction of households with each shock depends only on the fraction of households that had each shock the previous period. I deal first with versions of this economy that are stationary in the sense that, besides this idiosyncratic shock, there are no other time-varying features which affect the problem the agent faces.

\section{A. Stationary Economies: Steady States}

Households cannot borrow, but they can hold part of their endowment as inventory. Let $q>\beta$ denote the inventory technology, so that if $a_{t}$ goods are stored today, $a_{t+1}=\frac{a_{t}}{q}$ are obtained tomorrow. The agent then maximizes equation (1) subject to

$$
c_{t}=a_{t}+s_{t}-a_{t+1} q
$$

with $a_{0}$ given.

At this point, it is useful to deal with this problem by looking at its associated recursive formulation:

$$
\begin{aligned}
v(s, a) & =\max _{c, a^{\prime}} u(c)+\beta E\left\{v\left(s^{\prime}, a^{\prime}\right) \mid s\right\} \quad \text { s.t. } \\
c & =a+s-a^{\prime} q
\end{aligned}
$$

where I denote next period's variables by primes and where the solution is given by $a^{\prime}=g(s, a)$.

Note that at any point in time, there are a number of households that differ in their current luck $s$ and in their wealth $a$ (that somehow summarizes all their past luck). To describe this economy, I need a mathematical object that is well-suited to counting. This object is a measure. The key properties of measures are associated with the fact that they act as counting or weighting mechanisms. To do the mathematics properly, I review very summarily the concepts, although to understand the logic, the concepts are not really needed. 
- A $\sigma$-algebra is a family of subsets $\mathcal{A}$ of a certain set $A$, so that $\emptyset, A \in \mathcal{A}$ and $\mathcal{A}$ is closed under complementarity and under countable intersection.

- Borel $\sigma$-algebras are the $\sigma$-algebras generated by a family of open sets.

- A measure $x$ is a function $x: \mathcal{A} \rightarrow \mathbb{R}_{+}$such that $x(\emptyset)=0$, and if $B=\cup_{n=1}^{N} B_{N}, B_{n} \in \mathcal{A}$ with $B_{n} \cap B_{\hat{n}}=\emptyset$ for any pair $n, \hat{n} \in\{1, \cdot, N\}$, then $x(B)=\sum_{n=1}^{N} x\left(B_{n}\right)$. That is, the measure of disjoint sets should be the sum of the measure of each, they should be positive, and that's it. Note that probabilities are measures.

- A function $g$ is measurable with respect to $\{A, \mathcal{A}\}$ if the set $\{a: g(a) \leq c\}$ belongs to $\mathcal{A}$ for all $c \in \mathbb{R}$.

- A function $Q: A \times \mathcal{A} \rightarrow \mathbb{R}$ is a transition function if given $a \in A, Q(a, \cdot)$ is a probability measure and if given $B \in \mathcal{A}, Q(\cdot, B)$ is a measurable function.

- A measure $x^{*}$ is an invariant distribution with respect to the transition function $Q$ if for all $B \in \mathcal{A}, x^{*}(B)=\int_{A} Q(a, B) d x^{*}$.

Next I describe some of the crucial properties of the solution to equation (3). These properties are stated without proof, and the reader is referred to Huggett (1993) for details.

- Monotonicity and continuity are properties of the saving functions. Monotonicity only holds for certain types of Markov matrices $\Gamma$; in particular, it does hold for a two-state process.

- There are upper and lower bounds on the desired level of asset holdings, so that $a \in A=$ $[\underline{a}, \bar{a}]$ when $q>\beta$. The lower bound can arise from borrowing constraints or, as in the preceding literal interpretation, from technological feasibility. Alternatively, in economies where borrowing is feasible, this lower bound on assets can arise endogenously from the fact that the agent has to pay its debts in every state of the economy, which prevents the possibility of bankruptcy. In this case, the lower bound on assets is that level which requires that the worst possible endowment is used entirely to pay interest. If I denote this lower bound with $\underline{a}$ and $s_{1}$ is the worst earnings shock, I have that $\underline{a}=\frac{-s_{1}}{1-q}$.

- With the agent decision rule $g(s, a)$ and the Markov matrix $\Gamma$ that governs the law of motion of the idiosyncratic shock $s$, I can construct a transition function $Q(s, a, B)$ that describes the conditional probability for a type $(s, a)$ household to have a type in set $B \subset S \times A$ tomorrow. 
I use this transition to obtain the dynamic equation of the model that gives tomorrow's distribution of households $x^{\prime}$ as a function of today's distribution $x$ and the Markov chain $\Gamma$ by $x^{\prime}(B)=\int_{S \times A} Q(s, a, B) d x$. Note that a theorem which states that $x^{\prime}$ is, in fact, a probability distribution (or probability measure) is needed. I denote the updating operator with $T(x)$; that is, $x^{\prime}=T(x)$. I write

$$
T(x)=x^{\prime}(B)=\int_{S \times A} Q(s, a, B) d x
$$

for all $B \in \mathcal{A}$.

This model is useful if there are not many possible distributions of wealth of interest. In other words, I would like the model to generate endogenously some unique distribution of wealth so that the model has some tight predictions. There is good news in this respect.

As long as a certain monotone mixing condition is satisfied, it can be proved that there is a unique fixed point $x^{*}$ of equation (5). Furthermore, this point is the weak limit of successive applications of the operator associated to the above equation for any initial condition $x_{0}$. This mixing condition can be thought of, rather loosely, as the existence in this economy of both the American Dream and the American Nightmare. The condition requires that no matter how poor (rich) a household is, the probability that the household becomes one of the richest (poorest) is arbitrarily close to one, provided enough time passes.

Therefore, given a certain process $\{S, \Gamma\}$, there is a unique stationary distribution of wealth $x^{*}$ associated with it. In this economy, the stationary distribution of wealth corresponds to a steady state. The issue is how to find $x^{*}$, given $g$. Two possibilities are outlined below.

1. As with probabilities, distribution functions can be used to describe the measures. For this type of economy, I can use distribution functions over assets, but densities over shocks. Let $\left\{\Gamma_{n}^{*}\right\}_{n=1}^{N}$ be the invariant distribution associated to Markov matrix $\Gamma$. If today's distribution of the shock is given by $\Gamma^{*}$, so is tomorrow's. I approximate the distribution functions with, say, a piecewise linear function over some (very fine) grid of the asset space. To compute $x^{*}$, I use the following steps:

(a) Make a grid on the set $A$, such as $\left\{a_{1}, \cdots, a_{m}, \cdots, a_{M}\right\}$, where $a_{1}=\underline{a}$ and $a_{M}=\bar{a}$. Make sure that this grid is much finer than that used to compute $g$. The grid does not need to be equally spaced.

(b) Initialize an arbitrary $F^{0}$ over that grid. Note that $F^{0}$ is a matrix of dimension $N \times M$. 
For example, an initial distribution of wealth that is uniform can be represented by

$$
F_{n, m}^{0}=\frac{a_{m}-a_{1}}{\left(a_{M}-a_{1}\right)} \Gamma_{n}^{*}
$$

Alternatively, an initial distribution of wealth where all households hold the minimum wealth $\underline{a}$ can be represented by

$$
F_{n, m}^{0}=\frac{a_{M}-a_{1}}{\left(a_{M}-a_{1}\right)} \Gamma_{n}^{*}
$$

(c) Compute $g^{-1}\left(s_{n}, a_{m}\right)=\left\{a, a_{m}=g\left(s_{n}, a\right)\right\}$, the inverse of the decision rule (which is unique, given the established monotonicity of $g$ ). This process depends on how the decision rule $g$ was obtained. Typically, the process involves some type of interpolation, since $g$ is stored only on a finite set of points of the asset space.

$$
F_{n, m}^{1}=\sum_{\hat{n}=1}^{N} F^{0}\left(\hat{n}_{j}, g^{-1}\left(s_{\hat{n}}, a_{m}\right)\right) \Gamma\left(s_{n} \mid s_{\hat{n}}\right)
$$

Note that $F^{1}$ is written as a vector, while $F^{0}$ is written as a function. The reason is that $F^{1}$ is computed on a grid, while $F^{0}$ is evaluated in points that are not necessarily on the grid. To evaluate the value of $F^{0}$ outside the grid, interpolation is needed. Since the distribution function is approximated by a piecewise linear function, the interpolation is linear. Other interpolation techniques could also be used.

(d) Compare $F^{0}$ with $F^{1}$. If they are equal (up to required precision), stop; if not, update so that $F^{0}=F^{1}$, and repeat the process.

2. Alternatively, I can construct a sample of households and track them over time, as data collection agencies do. Let $I$ be the size of that sample (tens of thousands is an appropriate sample size). Then do the following:

(a) Initialize the sample by assigning a pair $\{s, a\}$ to each element $i$. This yields a sample distribution of wealth $\left\{\left\{s^{1}, a^{1}\right\}, \cdots,\left\{s^{i}, a^{i}\right\}, \cdots,\left\{s^{I}, a^{I}\right\}\right\}$.

(b) Compute a set of statistics from this sample, such as the average, standard deviation, percentiles, and so on, and denote them $H\left(\left\{s^{i}, a^{i}\right\}_{i=1}^{I}\right)$.

(c) Use the function $g$ and a random number generator to obtain $\left\{\left(s^{\prime}\right)^{i},\left(a^{\prime}\right)^{i}\right\}$ for all $i$, and compute its statistics $H$. 
(d) Continue this process until two consecutive sets of statistics are sufficiently close to each other. This means that consequent updates of the sample will not have distributional changes, and the sample is considered converged.

With the notation that I have used, $s$ denotes shocks to labor earnings. However, it is clear that the same logic can be used to denote many other shocks as well, such as shocks to health, marital status, entrepreneurial success, and so on. This notation can also be used to determine household characteristics that evolve deterministically, such as genetic type or age.

So far, there are no equilibrium considerations: all households live in isolation and increase or decrease their stockpiles of coconuts. However, I can actively introduce markets. I show two simple examples:

1. Households borrow from and lend to each other, and there is no storage [Huggett (1993)]. In this case, there is a market-clearing condition that did not exist before. Note that $q$ is the inverse of the gross interest rate. The market-clearing condition can be written as

$$
\int_{S \times A} a d x^{*}(q)=0
$$

where I have made explicit the dependence of the invariant distribution on the rate $q$ at which assets can be accumulated.

2. Assets take the form of real capital, and there is no borrowing and lending, $(\underline{a}=0)$. Let $f(K, L)$ be an aggregate production function, where aggregate employment $L$ is the total amount of efficiency units in the economy. The shock $s$ should be interpreted as a shock on the endowment of efficiency units of labor. That is, $L=\sum_{n=1}^{N} s_{n} \Gamma_{n}^{*}$, which does not change over time. Note that in this case, the key variable to be determined is aggregate capital, since the rate of return that households face is given by aggregate productivity of capital. This can be summarized by the following condition:

$$
f_{1}\left(\int_{S \times A} a d x^{*}(q), L\right)=\frac{1}{q}
$$

An important thing to note about the market interpretation of economies of this type is that not all possible prices $q$ are relevant: only those, if any, that clear markets. Therefore, those prices have to be found. The standard procedure for doing so is based on finding two prices - one 
at each side of the market-clearing condition - and then iterating within those two bounds until market-clearing occurs. A key property of those two prices is that $q>\beta$. Otherwise the household's problem does not have a bounded solution. Many sources study the problem of finding numerically the solution to one equation. See Judd (1991), for example.

I have already shown some of the procedures used to find the zero of an equation when values are found that give positive and negative values to the equation. To be sure that a zero exists, a sufficient condition is that the moments of $x^{*}(q)$ are continuous. Such conditions are typically satisfied for economies like this one. Theorem 12.13 in Stokey and Lucas (1989) requires the state space $\{S \times A\}$ to be compact, the transition probabilities $Q$ to be continuous in $q$, and each $q$ to have a unique stationary distribution $x^{*}(q)$.

So far, the model encompasses only the choices of consumption and savings. Some applications might require the inclusion of other variables.

- An hours-worked variable requires simply solving a slightly more complicated agent problem. Still, the same methods apply once the decision rules of the new problem are obtained. Unlike in methods designed to solve for the representative household of an economy, the possibility of corner solutions in hours worked might arise. That is, sometimes the members of the household choose to work zero hours. If that is the case and an Euler equation method is used to solve the agent's maximization problem, the typical procedure is to impose that hours worked are zero and then solve the intertemporal Euler equation under this restriction.

- A discrete choice variable, such as to work a fixed number of hours or not to work, to become an entrepreneur or a worker, or to go college or not, is much more difficult to solve. Note that because of the discreteness, information on the value function has to be stored, because the Euler equation is not enough to characterize the optimal choice. Moreover, note that the iterations on the value function do not necessarily return concave functions, and the decision rules are not necessarily continuous in many points of the state space. To see this, imagine the discrete choice of whether to work a fixed number of hours or not to work at all. For each $s$, there is a level of wealth - denote it $\hat{a}_{0}$ - high enough that the members of the household will choose to enjoy the leisure, while for lower levels of wealth they will choose to work. Note that for values of wealth slightly lower than $\hat{a}_{0}$, income can be considerably higher than at $\hat{a}_{0}$, since the household has almost the same capital income but a lot more labor income. Therefore, the savings decisions are discontinuous at $\hat{a}_{0}$. Take the case of a good enough shock 
so that the household is accumulating assets for $a<\hat{a}_{0}$. In this case, there is another level of assets - denote it $\hat{a}_{1}$ - so that $\hat{a}_{0}=g\left(s, \hat{a}_{1}\right)$. If $a \geq \hat{a}_{1}$ and if the shock does not change, the members of the household will choose not to work one period after, but if $a<\hat{a}_{1}$, the members of the household will choose to work the following period. Again, the same logic as before shows that there is another discontinuity in the savings function at $\hat{a}_{1}$. To deal with this type of problem, four approaches can be used.

1. Discretize the state space. This means that asset holdings can take only finitely many values. The optimal choice is just a finite maximization problem that can be solved with relative ease, typically taking advantage of the monotonicity, within the same discrete choice, of the decision rule. See, for example, Trick and Zin (1993).

2. Use lotteries. This means that households choose a probability of making the choice which makes the action space convex and allows the Euler equation approach to be used. However, this means that there is a contract between households, and a market-clearing condition is necessary.

3. Use shocks with a continuous support. In this case, the discontinuity that shows up one period before the household achieves the critical level might disappear. The reason is that one period before, the households cannot be sure of how their savings will relate to the critical discontinuity point.

4. Use continuous time models where the discontinuity problem is likely to disappear (at least the discontinuity that results from the rounding of the time periods). This method is still under development for this class of nonconcave problems.

Unlike in the environment where there is a storage technology, the model economy cannot be thought of as having measures changing over time. This would imply that market-clearing prices change every period, but that households solve their maximization problems under the assumption that prices are constant forever.

I now turn to situations where there are prices, but where there are time-varying distributions.

\section{B. Non-Steady-State Allocations}

I am interested in economies with a large number of households that face uninsurable, idiosyncratic uncertainty, but not necessarily in steady-state situations. For example, I might want to know the business cycle properties of this type of economy. Or, perhaps, I want to know the 
implications of a policy change, say, a tax change. I will run into a big problem. To see the problem, consider a simple real business cycle version of the economy where the asset is real capital. ${ }^{1}$ Households have $s$ units of labor and own assets that can be used as real capital in an aggregate production function given by

$$
Y_{t}=z_{t} f\left(K_{t}, L_{t}\right)
$$

where $Y_{t}$ is real output. Capital depreciates at rate $\delta$. Productivity shock $z_{t}$ follows a Markov process with transition $\Gamma_{z}$. Conditional on the aggregate shock $z_{t}$, the idiosyncratic shock $s_{t}$ is Markov, too. The joint process for these shocks can be written as $\Gamma\left(z^{\prime}, s^{\prime} \mid z, s\right)$. There are rental markets for labor and capital but, again, no insurance against bad realizations of the labor efficiency of each agent. Factor prices are marginal productivities. The problem of the agent in nonrecursive form can be written as

$$
\begin{aligned}
\max _{c_{t}, a_{t+1}} & E_{0}\left\{\sum_{t=0}^{\infty} \beta^{t} u\left(c_{t}\right)\right\} \quad \text { s.t. } \\
c_{t} & =a_{t}\left(1+r_{t}\right)+s_{t} w_{t}-a_{t+1}
\end{aligned}
$$

with $a_{0}$ given and where $w_{t}$ and $r_{t}$ are the rental prices of factors. To solve this problem, the household has to know the process that determines those prices. Those prices are determined by marginal productivities, which in turn depend on the aggregate quantities of the inputs. In this simple case, only aggregate capital matters. I could be tempted to write (as in the representative agent case) the following problem:

$$
\begin{aligned}
v(z, K, s, a) & =\max _{c, a^{\prime}} u(c)+\beta E\left\{v\left(z^{\prime}, K^{\prime}, s^{\prime}, a^{\prime}\right) \mid z, s\right\} \quad \text { s.t. } \\
c & =a(1+r(z, K))+s w(z, K)-a^{\prime} \\
K^{\prime} & =G(z, K)
\end{aligned}
$$

where $r(\cdot)$ and $w(\cdot)$ are functions that return the marginal productivity. This, however, is wrong. The problem is the function $G(\cdot)$. It is a problem because the pair $\{z, K\}$ is not, in general, a sufficient statistic for $K^{\prime}$ : tomorrow's capital $K^{\prime}$ depends on the whole distribution of wealth $x$. Depending on how wealth is distributed, aggregate capital will be different tomorrow, except in the case in which individual decision rules are linear in $a$, which is not the usual case. The appropriate

\footnotetext{
${ }^{1}$ Many economists do not like real business cycle models because they are representative agent, or Robinson Crusoe, worlds. Perhaps they will like this version more.
} 
problem is then

$$
\begin{aligned}
v(z, x, s, a) & =\max _{c, a^{\prime}} u(c)+\beta E\left\{v\left(z^{\prime}, x^{\prime}, s^{\prime}, a^{\prime}\right) \mid z, s\right\} \quad \text { s.t. } \\
c & =a(1+r(z, K))+s w(z, K)-a^{\prime} \\
K & =\int_{S \times A} a d x \\
x^{\prime} & =G(z, x) .
\end{aligned}
$$

The problem is that, unlike $a$ or the shocks $z$ and $s$, the measure $x$ is not a real number. On the contrary, it is an infinitely dimensional object, and keeping track of it requires a lot of storage. Moreover, computing the equilibrium requires not only solving the problem of the household, but also finding the function $G(z, x)$. Typically, the law of motion $G(z, x)$ of the economy is found by iterating on the space where this function belongs, but in this case, the appropriate space is the set of functions from an infinitely dimensional space into itself. The procedure involved is absolutely unmanageable.

There are three standard ways to get around this problem and still study economies with time-varying wealth distributions. I review them in order.

\section{The Distribution Is Not a State Variable}

The most obvious way to get around the problem is to deal with economies for which there are prices that do not depend on $x$, but on some exogenous feature, such as a shock. This can be done by using simple technologies or by using the government to impose prices exogenously. See Díaz-Giménez, Prescott, Fitzgerald, and Alvarez (1992), Ríos-Rull (1995), and Díaz-Giménez (1996). The latter procedure requires, of course, making sure that the government budget constraint is satisfied. In this approach, the distribution of wealth, $x$, is not a state variable of the household problem.

\section{Transition Between Steady States}

The second way to get around the problem is to look at the transition between steady states. This method was pioneered in Auerbach and Kotlikoff (1987). It is only useful for a deterministic economy that is in a steady state and then undergoes a change (completely unexpected), such as a change in technology or government policy. This method also requires stability of the steady state under the new parameters. The procedure is to assume that the economy converges in finite time 
to the new steady state in, say, 200 periods. This procedure can be thought of as a second-order difference equation with two end conditions (at opposite ends). This approach can be illustrated with the economy in Huggett (1993) after a change in the Markov matrix from $\Gamma$ to $\hat{\Gamma}$. The steps involved are the following.

1. Compute the steady-state distribution and equilibrium interest rates for both Markov matrices; that is, get $q(\Gamma), x(\Gamma)$ and $q(\hat{\Gamma}), x(\hat{\Gamma})$.

2. Note that the Euler equations for the household problem can be written as

$$
u^{\prime}\left(a_{t}+s-a_{t+1} q_{t}\right)=\frac{\beta}{q_{t}} \sum_{s^{\prime}} u^{\prime}\left(a_{t+1}-a_{t+2} q_{t+1}\right) \hat{\Gamma}\left(s^{\prime} \mid s\right) .
$$

3. Guess $q_{1}$.

4. Given $q_{1}$ and $x(\Gamma)$, solve for the $q_{2}$ that clears the credit market.

5. Update $x(\Gamma)$ to get $x_{1}$ using the decisions implied by $q_{1}$ and $q_{2}$.

6. Continue this process until $q_{200}$. Check whether $q_{200}$ and $x_{200}$ equal $q(\hat{\Gamma})$ and $x(\hat{\Gamma})$. If they do not, update the guess of $q_{1}$.

Alternatively, rather than think of $q_{200}$ as a function of $q_{1}$, think of $q_{1}$ as a function of $q_{200}$ and proceed backward. The backward procedure seems to be more efficient. [See Judd (1991) for a discussion of this point.]

\section{Partial Information}

The third way to get around the problem, which was introduced in Krusell and Smith (1995a) and also used in Castañeda, Díaz-Giménez, and Ríos-Rull (1997), is based on the logic that perhaps by using a little bit of information about $x$, households do almost as well as by using all the information in the distribution $x$ when predicting future prices. The type of information about $x$ that the agents use can be described generically as moments of the distribution. These moments include average wealth, standard deviation of wealth, and so on. They can also include such things as the share of the wealth held by the wealthiest $5 \%$ or even the Gini index. Mathematically, these moments are given by the integral of a certain function with respect to the measure $x$.

Under this approach, households are assumed to be boundedly rational in the sense that

they do not use all the information available to them. In this case, current and lagged values of 
$(z, x)$ are such information. They use only some bits and pieces of information of $x$, which I write as $\ell_{j}(x)$, where the index $j$ denotes the amount of information in the simple sense that it is the number of statistics of $x$ that is used. Function $\ell_{j}$ typically consists of integrals of functions of the state with respect to the measure $x$, such as the moments of $x$. For now, function $\ell_{j}$ must include sufficient statistics for factor prices, which are the reason the agent's problem is affected by the measure. Below I discuss the extension of this approach to the case when current prices depend on the current actions of the agents. Consider the following problem where, to simplify the notation, I define $y \equiv \ell_{j}(x)$ :

$$
\begin{aligned}
v\left(z, y, s, a ; h_{j}, \ell_{j}\right) & =\max _{c \geq 0, a^{\prime} \in[\underline{a}, \bar{a}]} u(c)+\beta E\left\{v\left(z^{\prime}, y^{\prime}, s^{\prime}, a^{\prime} ; h_{j}, \ell_{j}\right) \mid s, z\right\} \quad \text { s.t. } \\
y^{\prime} & =h_{j}(y, z) \\
c & =a(1+r(z, y))+s w(z, y)-a^{\prime}
\end{aligned}
$$

where $r(z, y)$ and $w(z, y)$ are functions for the factor prices that now depend on $y$. It is important to note that in this economy, factor prices only depend on the aggregate shock $z$ and on aggregate capital $K$. Note that I have indexed the value function both by function $\ell_{j}$, which returns the functions that serve as a proxy for measure $x$ as state variables, and by the forecasting function $h_{j}$, which returns the future values of those functions. The households that solve this problem are boundedly rational for two reasons. One is that when households predict the future values of prices, they do not use all the information at their disposal. They approximate the distribution by a finite set of its statistics, and they consider only the current-period values of those statistics to predict their future values. The other is that when households make forecasts of $y^{\prime}$, they act as if their forecasts were perfectly accurate, which they are not. Note that the forecasting errors are not random in the sense that if the distribution were used to forecast the value of $y^{\prime}$, there would be no prediction errors.

The solution to problem (22) is an optimal savings rule $g\left(z, y, s, a ; h_{j}, \ell_{j}\right)$, which, together with the process on $z$, generates a law of motion for the economy, $G\left(z, x ; h_{j}, \ell_{j}\right)$. Let $b_{j}\left(h_{j}, \ell_{j}\right)$ denote the best unbiased forecasting function of $y^{\prime}=\ell_{j}\left(x^{\prime}\right)$ within a certain parametric class $B$; that is, $\hat{y}^{\prime}=b_{j}\left(z, y ; h_{j}, \ell_{j}\right)$. Note that $b_{j}\left(\cdot, \ell_{j}\right)$ maps the set of $j$-dimensional functions in parametric class $B$ into itself and can be readily computed through long simulations. The procedure is simple: given $\left\{h_{j}, \ell_{j}\right\}$, solve the problem of the agent that obtains $g\left(z, y, s, a ; h_{j}, \ell_{j}\right)$. Then initialize a large sample of agents of dimension $I$ by giving agents a pair consisting of a shock $s$ and assets 
$a$, which yields $\left\{s_{i}^{0}, a_{i}^{0}\right\}_{i=1}^{I}$. From this sample, compute $\ell_{j}\left(x^{0}\right)$, which obtains $y^{0}$, and initialize the aggregate shock $z^{0}$. Use $\Gamma$ and $g$ to update the sample $\left\{s_{i}^{1}, a_{i}^{1}\right\}_{i=1}^{I}$, and compute $y^{1}$. Continue this procedure for a large number of periods (at least in the thousands). Drop the first half of the sample to avoid dependence on the initial conditions that were set arbitrarily. Use the values of the shocks $z$ and of the statistics $y$ in the second half of the sample to estimate a new forecasting function within parametric class $B .^{2}$ This will give the best predictor of $y^{\prime}$ within the parametric class $B$, the $b_{j}\left(h_{j}, \ell_{j}\right)$. Compare the values of $b_{j}\left(h_{j}, \ell_{j}\right)$ with $h_{j}$. If they are very similar, stop. If not, update $h_{j}$ using the information provided by $b_{j}$, and repeat the process. Typically, successive approximations are used to obtain $h^{*}\left(\ell_{j}\right)=b_{j}\left(h_{j}^{*}, \ell_{j}\right)$. Note that this fixed point is an essential part of any equilibrium in a model economy with boundedly rational households: when the households use linear predictor $h^{*}\left(\ell_{j}\right)$, their behavior generates a law of motion in which the best linear predictor is also $h^{*}\left(\ell_{j}\right)$.

Because a given distribution $x$ can be approximated by a large class of functions $\ell_{j}(x)$, and because forecasts can be made with different families of parametric forecasting functions $B$, I am not done yet. I have to single out an $\ell_{j}$ and a $B$.

Let $\left\{\ell_{j}(x)\right\}_{j=1}^{\infty}$ be a nested sequence of multivariate functions of dimension $j$. The larger the $j$, the more statistics it includes and, in the limit, all moments of $x$ are used. My objective is to find a multivariate function $\ell_{j}$ of small dimension $j$ with the property that economies in which the agents use functions of larger dimension to approximate the distribution display a similar equilibrium behavior. To determine whether the equilibrium behaviors of two economies are similar, a metric has to be chosen to compare those behaviors. There are several candidates for this metric. The distance between the stochastic realizations of the economies is one, and the distance between the optimal decision rules $a_{j}^{\prime}$ is another. A specific example is as follows: Choose a function $\ell_{j}$. Compute its associated equilibrium predictor $h^{*}\left(\ell_{j}\right)$. Compute a measure of its predictive accuracy, its $R$-squared or the variance of the one-period-ahead forecasting errors, for instance. ${ }^{3}$ Then choose a multivariate function of dimension $j+1$ such that $\ell_{j} \subset \ell_{j+1}$, compute the accuracy of the best forecasts of $\ell_{j}\left(x^{\prime}\right)$ conditional on $\left\{\ell_{j+1}(x), z\right\}$, and compare them with those implied by $h^{*}\left(\ell_{j}(x), z\right)$. If the difference is small, then the approximation to the equilibrium with boundedly rational agents

\footnotetext{
${ }^{2}$ Note that an obvious parametric class of forecasting functions is the set of linear forecasting functions. In this case, the estimation step becomes a simple linear regression.

${ }^{3}$ Continuity of the decision rules with respect to predicted values of $\ell_{j}\left(x^{\prime}\right)$ guarantees that small improvements in prediction imply small changes in actions. This property relates the metric defined on the accuracy of the predictor to the metric defined on the decision rule space.
} 
that use $\ell_{j}$ is satisfactory. Otherwise, a multivariate function of higher dimension must be used. One approach is to follow Krusell and Smith (1995a) by choosing $j=1$ and define $\ell_{1}(x)$ to be aggregate capital. This approximation turns out to work remarkably well in many environments.

To choose a family of forecasting functions, typically, the procedure is to start using linear forecasts and check whether those forecasts can be improved by using other types of forecasting functions, such as log-linear or simple polynomials. The choice of the type of forecasting function depends on the accuracyy of the forecasts. Linear and log-linear forecasts turn out to work quite well.

Note that this procedure works only when the function $\ell_{j}$ that is chosen includes sufficient statistics for prices. Decision rules $g\left(\cdot ; h_{j}, \ell_{j}\right)$ and the process for the shock $z$ are the only objects required to generate large samples of the actions of agents and, hence, of the statistics of the

distributions $y^{t}$ that are needed to find the required fixed point of the forecasting function $h_{j}$. However, if the prices are not ex-ante known statistics of the distribution, those prices have to be found before the behavior of agents can be simulated. Otherwise, the requirement that the simulated actions of agents clear the markets cannot be imposed. I turn now to how to modify the use of partial information in order to overcome this difficulty.

\section{Unknown Sufficient Statistics for Prices}

When current prices are not given by known statistics of the distribution, a two-step process can be used. The extra step ensures that markets clear and allows households to observe current prices before they act.

To illustrate how to deal with environments where the prices have to be dealt with, I consider two examples: a growth model with leisure and a growth model with both real assets and bonds. These examples are drawn from Krusell and Smith (1995a, 1995b). The first example is slightly simpler because agents forecast aggregate employment which, together with aggregate capital, is a sufficient statistic for factor prices. Determining the realized value of aggregate employment is simple: it is given by the actual actions of the agents. The second example is slightly more complex, because of the need to compute the equilibrium risk-free interest rate.

A Growth Model With Leisure Consider a version of the previous model in which households now care about leisure, so that the current utility function is given by $u(c, l)$. Aggregate factor prices now depend not only on aggregate capital, but also on the aggregate labor input $N$, which is not a 
known moment of the distribution because it depends on the current actions of all households. In the example without leisure, I looked for a set of moments $\ell_{j}$ and predictor $h_{j}$ for the values of the moments. In this example with leisure, I add a function that helps to predict aggregate employment and denote it $\xi_{j}(y)$. To avoid useless repetition, I restrict the predicting functions $h_{j}$ and $\xi_{j}(y)$ to be linear.

To illustrate how to adapt this procedure to the existence of prices that depend on the current actions of agents, it suffices to show the changes in the procedures to iterate on the value function and in the updating of the best linear predictors. Let $v_{0}\left(z, y, s, a ; h_{j}, \xi_{j}, \ell_{j}\right)$ be a value function for agents that predict future moments with $h_{j}$ and aggregate employment with $\xi_{j}$. Two problems have to be dealt with. One is how to obtain $v_{1}$, the value function given that next period's value is determined by $v_{0}$. Iterations on these value functions yield the time invariant solution to the household's problem $v\left(z, y, s, a ; h_{j}, \xi_{j}, \ell_{j}\right)$. The other problem is how to generate samples of the economy to obtain values for $y^{t}$ and $N^{t}$ when households use forecasting functions $h_{j}$ and $\xi_{j}$. Recall that this is a necessary step in order to iterate on the forecasting functions themselves.

To deal with the iterations on the value function, consider the following problem

$$
\begin{aligned}
\Omega^{0}\left(z, y, s, a, N ; h_{j}, \xi_{j}, \ell_{j}\right) & =\max _{c, l, a^{\prime}} u(c, l)+\beta E\left\{v^{0}\left(z^{\prime}, y^{\prime}, s^{\prime}, a^{\prime} ; h_{j}, \xi_{j}, \ell_{j}\right) \mid s, z\right\} \quad \text { s.t. } \\
y^{\prime} & =h_{j}(y, z) \\
c & =a(1+r(z, y, N))+s w(z, y, N)-a^{\prime}
\end{aligned}
$$

where $\Omega^{0}$ depends explicitly on aggregate employment $N$. It also depends on the forecasting function $\xi_{j}$, because $v^{0}$ depends on it too. The solution to this problem delivers decision rules for consumption $c_{\Omega}^{0}\left(z, y, s, a, N ; h_{j}, \xi_{j}, \ell_{j}\right)$, leisure $l_{\Omega}^{0}\left(z, y, s, a, N ; h_{j}, \xi_{j}, \ell_{j}\right)$, and savings $a_{\Omega}^{\prime 0}\left(z, y, s, a, N ; h_{j}, \xi_{j}, \ell_{j}\right)$. Now I can use the forecasting function $\xi_{j}$ to update the value function and reduce its arguments to the state variables by getting rid of aggregate employment.

$$
v^{1}\left(z, y, s, a ; h_{j}, \xi_{j}, \ell_{j}\right) \equiv \Omega^{0}\left(z, y, s, a, \xi_{j}(z, y) ; h_{j}, \xi_{j}, \ell_{j}\right)
$$

Iterations on this procedure yield the value function $v\left(\cdot ; h_{j}, \xi_{j}, \ell_{j}\right)$. I also obtain the decision rules, which I index by $\Omega$ because they depend separately on aggregate employment $N$. The decision rules are $c_{\Omega}\left(\cdot ; h_{j}, \xi_{j}, \ell_{j}\right), l_{\Omega}\left(\cdot ; h_{j}, \xi_{j}, \ell_{j}\right)$, and $a_{\Omega}^{\prime}\left(\cdot ; h_{j}, \xi_{j}, \ell_{j}\right)$.

The next step is to generate a large number of realizations of the economy to obtain values 
for $y^{t}$ and $N^{t}$ that will be used in the computation of the next best linear predictors and pricing function. To obtain a series for aggregate employment, I compute in each period the value for aggregate employment $N^{t}$ that solves

$$
N^{t}=\int_{S \times A}\left[1-l_{\Omega}\left(z^{t}, y^{t}, s, a, N^{t} ; h_{j}, \xi_{j}, \ell_{j}\right)\right] d x^{t}
$$

I use this value as the argument in the function $a_{\Omega}^{\prime}\left(\cdot ; h_{j}, \xi_{j}, \ell_{j}\right)$ to update the distribution

$x^{t+1}$. Recall that a large sample of agents is typically used to keep track of the distribution $x^{t}$. The use of the decision rules indexed by $\Omega$ ensures that agents' labor choices are consistent with the value for aggregate employment that they respond to. In other words, this two-step procedure allows households to react to actual prices, and not to just forecasted prices.

I use another illustration for the case in which households trade a riskless bond in addition to holding real capital. The approach is similar, provided the right transformation of the state variables at the individual level is used.

A Growth Model With Two Assets: Real Capital and Bonds Consider a stochastic growth model with two assets: real capital (stocks) and a one-period zero coupon bond that should be in zero net supply (bonds). The rate of return on stocks is the marginal productivity of capital. The rate of return on bonds is the inverse (minus one) of whatever bond price clears the market. The market-clearing price for bonds needs to be found in order to generate large samples of the economy and then update the forecasting functions. Let $d$ denote the total resources available to a household whose members do not work at all: that is, the sum of capital income, real capital, and bond holdings. Let $a^{\prime}$ denote the household's holdings of real capital between this period and the next, and let $b$ denote the household's holdings of bonds between this period and the next. It is easy to see that I do not have to know the portfolio composition of the households; all relevant information is embodied in the variable $d$. Let $q$ be the current price of the bond. The distribution of agents across $\{d, s\}$, again denoted $x$, is the state variable I want to avoid in the problem of the agents. Note that the current aggregate shock $z$ affects the current distribution $x$. Let $\ell_{j}$ denote a set of statistics that the households use to summarize the distribution $x$, with $y=\ell_{j}(x)$. Let $h_{j}$ be a forecasting function of those statistics. Finally, let $\xi_{j}(y)$ be a forecasting function for the price $q$. Again, let $v_{0}\left(z, y, s, a ; h_{j}, \xi_{j}, \ell_{j}\right)$ be a value function of an agent that forecasts with $h_{j}$ and $\xi_{j}$. Consider the 
following problem:

$$
\begin{aligned}
\Omega^{0}\left(z, y, s, d, q ; h_{j}, \xi_{j}, \ell_{j}\right) & =\max _{c, b, a^{\prime}} u(c)+\beta E\left\{v^{0}\left(z^{\prime}, y^{\prime}, s^{\prime}, d^{\prime} ; h_{j}, \xi_{j}, \ell_{j}\right) \mid s, z\right\} \quad \text { s.t. } \\
y^{\prime} & =h_{j}(y, z) \\
c & =d+s w(z, y)-a^{\prime}-b q \\
d^{\prime} & =a^{\prime}\left(1+r\left(z^{\prime}, y^{\prime}\right)\right)+b
\end{aligned}
$$

where the associated procedure to update the value function is given by

$$
v^{1}\left(z, y, s, a ; h_{j}, \xi_{j}, \ell_{j}\right) \equiv \Omega^{0}\left(z, y, s, a, \xi_{j}(z, y) ; h_{j}, \xi_{j}, \ell_{j}\right)
$$

Iterations on this procedure yield the time-independent value function $v\left(z, y, s, a ; h_{j}, \xi_{j}, \ell_{j}\right)$ and decision rules $c_{\Omega}\left(\cdot ; h_{j}, \xi_{j}, \ell_{j}\right), b_{\Omega}\left(\cdot ; h_{j}, \xi_{j}, \ell_{j}\right)$, and $a_{\Omega}^{\prime}\left(\cdot ; h_{j}, \xi_{j}, \ell_{j}\right)$.

The next step is to generate a large number of realizations of the economy to obtain values for $y^{t}$ and $q^{t}$ that will be used in the computation of the next best linear predictors. To obtain a series for the price of the bond, I compute in each period the bond price that clears the market: that is, the value of $q^{t}$ that solves

$$
0=\int_{S \times D} b_{\Omega}\left(z^{t}, y^{t}, s, d, q^{t} ; h_{j}, \xi_{j}, \ell_{j}\right) d x^{t}
$$

I use this value as the argument in the functions $a_{\Omega}^{\prime}\left(\cdot ; h_{j}, \xi_{j}, \ell_{j}\right)$ and $b_{\Omega}\left(\cdot ; h_{j}, \xi_{j}, \ell_{j}\right)$ along with the law of motion of the aggregate shock $z$ to update the distribution $x^{t+1}$, and, consequently, of its moments $y^{t+1}$. With these elements, I am now in a position to upgrade the prediction functions, and I can proceed with the method described above to compute the equilibria of the economy with boundedly rational agents.

Krusell and Smith (1995a, 1995b), which developed this type of approach with models in which the distribution of wealth across agents is a state variable, found that typically the first moment of the wealth distribution suffices to produce an extremely high quality approximation, with the forecasting functions being linear or loglinear.

This discussion concludes the consideration of economies in which the distribution of agents across individual states changes over time. I now turn to the study of overlapping generations economies. 


\section{Overlapping Generations Economies}

These are economies in which the heterogeneity of households has the added dimension of age, and thus I will refer to them as agents. The term overlapping generations refers to the fact that agents are born in each period, and after a certain number of years, they die. There is another type of model, a sort of hybrid between infinitely lived agent models and overlapping generations models, in which new agents are born in each period and then face a constant probability of death. In these models, aging, or death, is stochastic, while in the overlapping generations models, aging is deterministic and agents die with probability one once they reach a certain age. These hybrid models are sometimes referred to as models with exponential population and can be analyzed with methods very similar to the ones described here.

I start by describing the environment for an overlapping generations economy embodied in a standard neoclassical growth model. The demographics are very simple: there is no population growth and no early death. Each period, a large number, a continuum that I normalize to one, of agents are born. Agents live for $I$ periods, and then they die. So at any point in time, there is a set of agents indexed by $i \in \mathcal{I}=\{1, \cdots, I\}$. Note that I do not keep track of the age in the same way that is done with people, since upon birth, agents have age one. These agents have standard preferences over consumption at different ages, which can be represented by a time-separable utility

function with age-specific weights $\beta_{i}$, given by $\sum_{i \in \mathcal{I}} \beta_{i} u\left(c_{i}\right)$. I assume that at each age, agents are endowed with $\epsilon_{i}$ efficient units of labor that they supply inelastically. The problem of an agent born in period $t$ is given by

$$
\begin{array}{ll}
\max _{c_{i t}, a_{i+1, t}} & \sum_{i \in \mathcal{I}} \beta_{i} u\left(c_{i t}\right) \quad \text { s.t. } \\
& a_{1 t}, a_{I+1, t}=0 \\
& c_{i t}=a_{i t}\left(1+r_{t+i-1}\right)+\epsilon_{i} w_{t+i-1}-a_{i+1, t}
\end{array}
$$

where $a_{i t}$ denotes the wealth of an age $i$ agent born in period $t$, and where $r_{\tau}$ and $w_{\tau}$ denote the rental prices of capital and labor in period $\tau$. The first constraint states that the agent is born with zero wealth and that upon death, the agent cannot have debts. The second constraint is the standard budget constraint. Note that in this specification of the model, agents are allowed to borrow, because there is no nonnegativity constraint for the assets. Typically, equilibrium for this economy is defined as a sequence of factor prices and allocations such that, given factor prices, the 
allocations are utility-maximizing and the markets clear. However, this definition is generally not very useful for computational purposes. I turn now to the discussion of steady states, because it is the simplest context in which to study this class of economies.

\section{A. Steady States}

A steady state is an equilibrium that does not change over time. In this context, a steady state consists of a pair of rental prices for capital and labor and age profiles for assets and consumption. Computation of a steady state for this economy requires solving one equation with one unknown in the aggregate capital-to-labor ratio, but each evaluation of the equation requires a solution to a maximization problem that is effectively a two-boundary difference equation. The bounds are those implied by equation (37), and the equation is the first-order condition of problem (36). The standard reference for this problem is Auerbach and Kotlikoff (1987). The following steps are involved.

1. Compute the aggregate labor input $L=\sum_{i \in \mathcal{I}} \epsilon_{i}$.

2. Initialize aggregate wealth $K^{0}$. Recall that in this economy, aggregate wealth is the sum of the assets held by agents of all ages.

3. Given $K^{0}$ and $L$, use marginal conditions to obtain a pair of factor prices $\left\{r^{0}, w^{0}\right\}$.

4. Given $\left\{r^{0}, w^{0}\right\}$, solve the problem of the agent, thereby obtaining $\left\{a_{i}\right\}_{i \in \mathcal{I}}$. To do this, obtain a second-order difference equation in assets from the first-order conditions of (36). When utility is of the constant relative risk aversion (CRRA) class, $u(c)=\frac{c^{1-\sigma}}{1-\sigma}$. The difference equation is of the form

$$
a_{i+2}=\left[a_{i+1}(1+r)+w \epsilon_{i+1}\right]-\left[\frac{\beta_{i+1}}{\beta_{i}}(1+r)\right]^{\frac{1}{\sigma}}\left[a_{i}(1+r)+w \epsilon_{i}-a_{i+1}\right]
$$

Solving this equation for $a_{1}=a_{I+1}=0$ is relatively easy. A simple, but perhaps not very efficient, way of doing so is to think of the difference equation (39) as a single equation that for each value of $a_{2}$ yields a value for $a_{I+1}$. Then solve such single equation. Perhaps a more efficient way of solving the difference equation is to go backward. Rewrite (39) so that $a_{i}$ is a function of $a_{i+1}$ and of $a_{i+2}$. Think of successive applications of (39) as yielding a function that, given $a_{I}$, returns $a_{1}$, and solve for a zero of such a function. Again, see Judd (1991) for a discussion of why the backward procedure is more efficient. 
5. Obtain a new value for aggregate wealth $K^{1}$ as the sum of the assets obtained from the previous step.

6. If $K^{1}=K^{0}$, stop. If not, upgrade the guess for $K^{0}$. The most efficient way to upgrade $K^{0}$ is to do it in the context of a solution algorithm, to find the zero of one single equation. That equation is $K^{0}-K^{1}\left(K^{0}\right)$.

I now review approaches for situations outside the steady state.

\section{B. Non-Steady-State Allocations}

Just as I am interested in the model with infinitely lived agents with idiosyncratic shocks, I am also interested in the behavior of the overlapping generations model outside a steady state. Also, as in the previous class of models, there are three ways of getting around the problem of having measures as state variables in the agent's problem: having constant prices, computing the transition between steady states, and looking at recursive equilibria. Computationally, under certain conditions, recursive equilibria with fully rational agents can be approximated. Otherwise, compute the equilibria of a similar economy with approximating, not fully rational, agents as it was done in the model with infinitely lived agents.

\section{Constant Factor Prices}

Again, one simple mechanism that prevents the distribution of wealth from affecting the decision of the agents is to make the factor prices, which provide the channel through which the state of the economy affects individual decisions, independent of the distribution. The easiest form of doing this is to assume the factor prices constant. To be able to do this, the small-country assumption should be made. This assumption states that world interest rates cannot be affected by anything that happens in the small country, and it prevents the agents from having to perform complicated calculations to predict interest rates. Real wages in this context become just a matter of normalization of units, and their value is irrelevant. For stochastic environments, the small-country assumption can also be used, as long as the structure of the shocks does not interact with the decision of the agents to determine prices. Whether this approach is justifiable or not depends, of course, on the question being asked. 


\section{Transition Between Steady States}

In exactly the same fashion as in economies with infinitely lived agents subject to uninsurable idiosyncratic risk, overlapping generations economies can be studied by looking at the transition between steady states. In fact, it is in this type of economy that this method was pioneered in Auerbach and Kotlikoff (1987). The approach is identical to that described in the previous section.

\section{Recursive Stochastic Equilibria}

In this section, I show how to define and compute equilibria recursively for stochastic versions of overlapping generations economies. The key references are Ríos-Rull (1992, 1994a, 1995, 1996).

To illustrate how to implement a stochastic version of the overlapping generations model, I look at a real business cycle version of the basic model. There is a neoclassical production function, $f(K, L)$, which is affected by a multiplicative shock $z \in Z=\left\{z_{1}, z_{2}, \ldots, z_{N_{z}}\right\}$. This shock follows a Markov process with transition matrix $\Gamma\left(z^{\prime} \mid z\right)$, and the shock is observed at the beginning of the period. Output can be used either for consumption in the same period that production takes place or for increasing the capital stock in the next period. Capital depreciates at rate $\delta$, and for notational simplicity, I assume that undepreciated capital can also be used for consumption. If I denote next period's variables by primes, all this can be written as

$$
\sum_{i=1}^{I} c_{i} x_{i}+K^{\prime}=z F(K, L)+(1-\delta) K
$$

where aggregate factors $K$ and $L$ are obtained as before by adding the assets and the endowment of efficiency units of labor across all cohorts.

Before I make the problem of the individual agent explicit, it is important to note that aggregate shocks to productivity present the possibility of state-contingent markets which allow for trades that pay only in the event of specific realizations of the shock. Ríos-Rull (1994a) studies the importance of the existence of these markets and concludes that they are quantitatively unimportant. For this reason, I am going to abstract from them. ${ }^{4}$ Consequently, the allocations I obtain will not be Pareto optimal. Note also that in this model, the economy-wide state is the pair $\{z, A\}$ : the shock and the distribution of assets across age groups. Because all agents within the same cohort are identical, $A \in \mathbb{R}^{I-1}$. Recall that agents are born with zero wealth, which implies that I only

\footnotetext{
${ }^{4}$ See Ríos-Rull (1995) for a description of the recursive implementation of this type of economy with complete markets and leisure.
} 
have to keep track of the wealth of agents of ages 2 to $I$. Also note that as in the previous section, aggregate capital $K$ is a sufficient statistic for prices in this period, but it is not a sufficient statistic for aggregate capital in the next period. The distribution of assets has be known to predict aggregate capital next period. Given these considerations, the individual state is $\{z, A, a\}$, where $a \in \mathbb{R}$ is the individual wealth. Denote by $v_{i}(z, A, a)$ the residual utility of an age $i$ agent with wealth $a$ when the aggregate state is given by $\{z, A\}$. Then the agent's problem is

$$
\begin{aligned}
v_{i}(z, A, a) & =\max _{c, a^{\prime}} u(c)+\beta_{i+1} \sum_{z^{\prime} \in Z} v_{i+1}\left(z^{\prime}, A^{\prime}, a^{\prime}\right) \Gamma\left(z^{\prime} \mid z\right) \quad \text { s.t. } \\
c & =a(1+r(z, A))+\epsilon_{i} w(z, A)-a^{\prime} \\
A^{\prime} & =G(z, A)
\end{aligned}
$$

with the solution given by $a_{i+1}=g_{i}(z, A, a)$, where $r(z, A)$ and where $w(z, A)$ are the marginal productivities of capital and labor that depend on aggregate capital, which is obtained by adding the assets of all the households, and where $G(z, A)$ is the evolution of the distribution of assets over time. Note that $G: Z \times \mathbb{R}^{I-1} \rightarrow \mathbb{R}^{I-1}$. Also note that $G$ is unknown and is part of the equilibrium process, and it should be computed simultaneously with the solution to the agent's problem. The equilibrium condition of this model is that agents are representative of their cohort:

$$
g_{i}\left(z, A, A_{i}\right)=G_{i+1}(z, A)
$$

In this context, a feasible computational procedure to solve for the equilibrium of this economy is to use linear quadratic approximations to the current return as a function of the states and the decisions and to follow the standard practices in the real business cycle as described, for example, in Hansen and Prescott (1995). The following is a summary description of this process.

1. Compute the steady state of the model economy when the shock has been set to its unconditional mean. I have already described how to do this computation.

2. Compute a set of linear quadratic approximations to the current return around the steady state. This step yields a set of $I N_{z}$ symmetric matrices $R_{i z}$ of dimension $I+3$, where the quadratic form $\left(1, A, a, a^{\prime}\right) R_{i z}\left(1, A, a, a^{\prime}\right)^{T}$ gives the current utility of an agent when the state is $\{z, A, a\}$ and the agent's choice is $a^{\prime}$. These matrices can be obtained by numerical computation of the Hessian. Again, see Hansen and Prescott (1995) for details. 
3. Initialize $v_{i z}^{0}$ for $i=\{1, \cdots, I\}, z=\left\{z_{1}, \cdots, z_{N_{z}}\right\}$.

4. Solve the following problem:

$$
\max _{a^{\prime}} R_{i z}\left(A, a, a^{\prime}\right)+\beta_{i+1} \sum_{z^{\prime} \in Z} v_{i+1, z^{\prime}}^{0}\left(z^{\prime}, A^{\prime}, a^{\prime}\right) \Gamma\left(z^{\prime} \mid z\right)
$$

with $v_{I+1}^{0}=0$.

Note that to avoid very cumbersome notation, I have written the current and future returns as if they were functions when they are quadratic forms. Also note that the solution is a set of linear functions $a_{i}^{\prime}=\varphi_{i z}\left(A, a, A^{\prime}\right)$. Finally, note that the $\varphi^{\prime}$ s are not the decision rules, since these functions depend on the next-period distribution of wealth.

5. Solve for the fixed point of the asset accumulation equation: when agents are equal to those representative of their age, they make the same choice as the other members of their cohort. This implies the system (which I write compactly by piling up the $i$ individual terms in each row)

$$
A^{\prime}=\varphi_{z}^{01} A+\varphi_{z}^{02} A^{\prime}
$$

where $\varphi_{z}^{01}$ are those terms of $\varphi_{i z}^{0}$ that affect current variables with the term that depends on $a$ added to the corresponding term that multiplies $A_{i}$.

6. Now obtain the economy-wide law of motion of asset holdings from the $\varphi$ by

$$
A^{\prime}=G_{z}^{1} A=\left(I-\varphi_{z}^{2}\right)^{-1} \varphi_{z}^{1} A
$$

7. Update the value functions by substituting the law of motion of the asset distribution $G^{1}$ and the individual decisions $\varphi$ in equation (41) to obtain $v_{i z}^{1}$.

8. Compare $v_{i z}^{0}$ with $v_{i z}^{1}$. If they are equal, stop. Otherwise, update $v_{i z}^{0}$ (perhaps by letting $v_{i z}^{0}=v_{i z}^{1}$ ), and go again to step 4 .

The procedure just outlined has the advantage that it lets the agents use all the relevant information to predict the future distribution of wealth. The procedure has, however, the disadvantage that it is very time consuming when the number of periods is large. This procedure has the property that the quality of the approximations is not necessarily very good when the economy is not too close to 
the steady state. Recall that the current objective is a linear quadratic approximation around the steady state.

\section{Other Approaches to Approximating Recursive Equilibria}

An alternative procedure for computing recursive equilibria is to do exactly as in the previous section: let the agents approximate the distribution with some of its moments and use sample realizations to update the predicting rule. This alternative procedure avoids the problem of the previous procedure when the economy is not close to the steady state. Another advantage is that it can be applied to economies in which not all agents within the same age group are identical. I use the same notation as in Section 2 and allow for both idiosyncratic and aggregate shocks. Let $x$ denote a measure over the individual states, which in this case can be written as $\{I \times S \times A\}$. In this context, $x(i, S, A)$ is the measure of agents with age $i$. The demographic evolution of the population can be easily implemented into the law of motion of $x$. Let $\ell_{j}$ be a set of statistics of $x$. The set must include aggregate capital and whatever is needed to compute current prices, but it may also include other features, such as the dependency ratio, the variance of wealth, and so on. ${ }^{5}$ Again, I define $y \equiv \ell_{j}(x)$. Now I can write the problem of the age $i$ agent as

$$
\begin{aligned}
& v_{i}\left(z, y, s, a ; h_{j}, \ell_{j}\right)=\max _{c \geq 0, a^{\prime} \in[\underline{a}, \bar{a}]} u(c)+\beta E\left\{v\left(z^{\prime}, y^{\prime}, s^{\prime}, a^{\prime} ; h_{j}, \ell_{j}\right) \mid s, z\right\} \quad \text { s.t. } \\
& y^{\prime}=h_{j}(y, z) \text {. } \\
& c=a(1+r(z, y))+s w(z, y)-a^{\prime}
\end{aligned}
$$

where, again, $h_{j}$ is the predictor function the agents use. Let the solution to this problem be $g_{i}\left(z, y, s, a ; h_{j}, \ell_{j}\right)$, and let the associated law of motion of the economy be $x^{\prime}=G\left(z, x ; h_{j}, \ell_{j}\right)$. Let $b_{j}\left(h_{j} ; \ell_{j}\right)$ denote the best linear, unbiased forecasting function of $y^{\prime}=\ell_{j}\left(x^{\prime}\right)$; that is, $\hat{y}^{\prime}=b_{j}\left(h_{j}, \ell_{j}\right) y$. Note that $b_{j}\left(\cdot, \ell_{j}\right)$ maps the set of linear $j$-dimensional functions into itself and can be readily computed through long simulations. Successive approximations can be used to obtain a fixed point in the space of predictor functions, $h^{*}\left(\ell_{j}\right)=b_{j}\left(h_{j}^{*}, \ell_{j}\right)$. Note that this fixed point is an essential part of any equilibrium in a model economy with boundedly rational agents: when agents use linear predictor $h^{*}\left(\ell_{j}\right)$, their behavior generates a law of motion in which the best linear predictor is also $h^{*}\left(\ell_{j}\right)$. Now I can use the same logic as in Section 2 to choose an appropriate set of statistics $\ell_{j}$.

\footnotetext{
${ }^{5}$ See above for the case in which there is no moment of the wealth distribution that is a sufficient statistic for current prices.
} 
Among the possible advantages of this procedure is the fact that I can look at economies with a very large number of periods and, therefore, ages of agents. Then rather than compute $I$ different decision rules with the associated large computational costs, I can make the decision rule depend parsimoniously on the age $i$ of the agent. This will avoid the linear quadratic approach, which will make this procedure more useful.

\section{Other Issues in Overlapping Generations Models}

To conclude the section on overlapping generations models, let me describe some issues that have been addressed with the aid of these models. Auerbach, Kotlikoff, Hagemann, and Nicoletti (1989), Ríos-Rull (1994b), Storesletten (1994), and Cubeddu and Ríos-Rull (1996) look at how demographic changes (for example, population aging, drops in fertility, changes in immigration policy, and increased incidence of divorce and out-of-wedlock births) affect the long-term behavior of savings. Imrohoroğlu, Imrohoroğlu, and Joines (1994) and Huang, Imrohoroğlu, and Sargent (1997) look at properties of the U.S. Social Security system.

\section{Dynamic Voting Models}

Next I study economies where, in equilibrium, the policies are determined jointly with the allocations through a political mechanism. Rather than look at a very general model, I will look at an example where the policy is a proportional income tax where the proceeds are redistributed lump-sum to all agents. The issue is the level of taxation and the associated values for the economic variables. In this world, poor households want high income taxes, while rich households want low (actually negative) income taxes. The question of interest is how much redistribution occurs, and how economic and institutional variables shape the amount of redistribution. Note that because taxation is distortionary, complete confiscation cannot be optimal. Finally, a constitution has to be in place that determines the set of feasible policies, how often they are reassessed, and how individual preferences over policies translate into a policy for the economy. In the example that I pose, every period the tax rate for the following period is chosen by majority rule. The ideas that I develop have a much wider applicability: for example, they can be applied to study the behavior of a government (altruistic or not) that cannot commit itself to future actions. By construction, the equilibrium policies that I obtain are time consistent. The main references are Krusell and Ríos-Rull (1994) and Krusell, Quadrini, and Ríos-Rull (1996, 1997). 
I define equilibrium recursively, which means I am interested in Markov perfect equilibria. I define economic equilibrium given that policy follows a certain arbitrary function $\Psi$. Then I turn to the fixed-point problem that generates a $\Psi$.

\section{A. The Environment}

The environment is once again the standard growth model. There are a finite number of infinitely lived agents of type $i \in \mathcal{I}=\{1, \cdots, I\}$, where $x_{i}$ is the fraction of each type. Types differ in the amount of initial assets $a_{i}$ and in their (constant) endowment of efficiency units of labor $\epsilon_{i}$. In this environment, agents care about consumption and leisure. Preferences are standard and are given by $\sum_{i} \beta^{t} u\left(c_{t}, l_{t}\right)$. There is also a standard neoclassical production function $f(K, L)$, and capital depreciates at rate $\delta$. Agents' assets are in the form of capital that they rent to competitive firms.

There is a government that taxes income proportionally and distributes the proceeds in a lump-sum fashion to all agents. For now, I assume that the tax rate of the current period was determined in the past and that the tax rate for the following period is determined this period through a function $\Psi$ of the aggregate state of the economy. Note that there is no uncertainty in this environment; therefore, all households of the same type will always be identical. This lack of uncertainty guarantees that the distribution of wealth can be represented by $A \in \mathbb{R}^{I}$. The aggregate state is thus the pair $\{A, \tau\}$, where $\tau$ is the tax rate inherited from the previous period.

\section{B. Economic Equilibrium}

I start by considering the equilibrium of an economy in which policy is set by an arbitrary policy function $\Psi$. I refer to this as the economic equilibrium.

I can write the maximization problem of household $i$ in its dynamic programming formulation as follows:

$$
\begin{aligned}
v_{i}(A, \tau, a ; \Psi) & =\max _{c, l, a^{\prime}} u(c, l)+\beta v_{i}\left(A^{\prime}, \tau^{\prime}, a^{\prime} ; \Psi\right) \quad \text { s.t. } \\
a^{\prime} & =a+\left[a r(K / L)+w(K / L)(1-l) \epsilon_{i}\right](1-\tau)+T-c \\
T & =\tau(f(K, L)-\delta K) \\
\tau^{\prime} & =\Psi(A, \tau) \\
A^{\prime} & =G^{A}(A, \tau ; \Psi)
\end{aligned}
$$




$$
\begin{aligned}
K & =\sum_{i} x_{i} A_{i} \\
L & =G^{L}(A, \tau ; \Psi)
\end{aligned}
$$

where $K$ and $L$ are aggregate capital and aggregate labor, $T$ is the per capita transfer, $r$ and $w$ are the factor pricing functions that depend only on the capital-to-labor ratio, $G^{A}$ is the economy-wide law of motion for asset holdings, and $G^{L}$ is the aggregate labor input function. Note that these functions are indexed by $\Psi$, since the behavior of the economy depends on the policy rule that determines the evolution of taxation. The solution to this problem is a pair of functions for the next period's asset holdings $a^{\prime}=g_{i}(A, \tau, a ; \Psi)$ and leisure $l=\ell_{i}(A, \tau, a ; \Psi)$ of the type $i$ households.

A recursive competitive equilibrium, given the function $\Psi$, is a standard concept. It is a set of functions $\left\{G^{A}, G^{L},\left\{h_{i}, \ell_{i}\right\}_{i \in \mathcal{I}}\right\}$ such that

$$
\begin{aligned}
G_{i}^{A}(A, \tau ; \Psi) & =g_{i}\left(A, \tau, A_{i} ; \Psi\right) \\
G^{l}(A, \tau ; \Psi) & =\sum_{i} x_{i}\left(1-l_{i}\left(A, \tau, A_{i} ; \Psi\right)\right) \epsilon_{i}
\end{aligned}
$$

for all $\tau, A$, and $i$. These conditions represent the fixed-point problem of the recursive equilibrium formulation; that is, they require that the optimal laws of motion of the individual households reproduce the aggregate laws of motion the households perceive when solving their decision problems. The economic equilibrium can be easily computed by standard methods. In particular, it can be solved by linear quadratic approximations. Later, I will discuss where I should do the approximation, what a steady state is, and how to find it.

A steady state here is a pair $\{A, \tau\}$ such that

$$
\begin{aligned}
A & =G_{i}^{A}(A, \tau ; \Psi) \\
\tau & =\Psi(A, \tau)
\end{aligned}
$$

Note that, in general, there will be more than one unique steady state. It is more like an $(I-1)$-dimension set of steady states. To see why, imagine a $\Psi$ that delivers a constant tax rate and a version of the economy in which all households have the same efficiency and in which leisure is not valued. It is immediate to see that in such a version, any $\left\{A_{i}\right\}_{i=1}^{I}$ with the property that $f^{\prime}\left(\sum_{i} x_{i} A_{i}\right)(1-\tau)=\beta^{-1}$ is a steady state. Such a set of $A_{i}^{\prime}$ 's is an $(I-1)$-dimensional subspace of the state space. 
In the above economic equilibrium, taxes are given by $\Psi$ at every point in time. In order to define the political equilibrium, I need to also consider economic equilibria where taxes are set slightly differently: that is, one-period deviations in tax policies. I need to be able to assess the utility that alternative policies yield. Consider that $\tau^{\prime}$ is set arbitrarily but that all tax rates at later dates are determined by the function $\Psi$. These are the situations that the household needs to think through when contemplating a current vote. Let $\tilde{H}$ and $\tilde{G}$ denote the law of motion of assets and the total labor supply function, respectively, for these deviations; these functions have $\tau^{\prime}$ as an argument. Therefore, consider the following problem for a given agent of type $i$ who has wealth $a$ :

$$
\begin{aligned}
\tilde{v}_{i}\left(A, \tau, \tau^{\prime}, a ; \Psi\right) & =\max _{c, l, a^{\prime}} u(c, l)+\beta v_{i}\left(A^{\prime}, \tau^{\prime}, a^{\prime} ; \Psi\right) \text { s.t. } \\
a^{\prime} & =a+\left[a r(K / N)+w(K / N)(1-l) \epsilon_{i}\right](1-\tau)+T-c \\
T & =\tau(F(K, N)-\delta K) \\
A^{\prime} & =\tilde{G}^{A}\left(A, \tau, \tau^{\prime} ; \Psi\right) \\
K & =\sum_{i} x_{i} A_{i} \\
N & =\tilde{G}^{L}\left(A, \tau, \tau^{\prime} ; \Psi\right) .
\end{aligned}
$$

In this problem - where the next period's tax rate is given, as opposed to determined by $\Psi$ - it is important to note that the next period's value function is given by the solution to problem (51). The decision rules for problem (56) are given by $a^{\prime}=\tilde{g}_{i}\left(A, \tau, \tau^{\prime}, a ; \Psi\right)$ and $l=\tilde{l}_{i}\left(A, \tau, \tau^{\prime}, a ; \Psi\right)$. The equilibrium conditions for the deviation problem are $\tilde{G}_{i}^{A}\left(A, \tau, \tau^{\prime} ; \Psi\right)=\tilde{g}_{i}\left(A, \tau, \tau^{\prime}, A_{i} ; \Psi\right)$ and $\tilde{G}^{L}\left(A, \tau, \tau^{\prime}\right)=\sum_{i} x_{i}\left(1-\tilde{l}_{i}\left(A, \tau, A_{i} ; \Psi\right)\right) \epsilon_{i}$ for all $\tau, \tau^{\prime}, A$, and $i$. These functions can be readily computed by standard methods. Note that the computation does not involve iterations, because once the function $v_{i}$ is known, calculation of functions $\tilde{v}_{i}$ and $\tilde{G}$ is a one-step procedure.

\section{Politico-Economic Equilibrium}

The objects I have computed so far are the economy's law of motion for the distribution of wealth $G^{A}(\cdot, \cdot ; \Psi)$, household $i$ 's utility when policy is given by $\Psi v_{i}(\cdot, \cdot, \cdot ; \Psi)$, the economy's law of motion for the distribution of wealth when the tax rate of the next period is given by $\tau^{\prime}$ and the economy reverts to using $\Psi$ to set the policies $\tilde{G}^{A}(\cdot, \cdot, \cdot ; \Psi)$, and agent $i$ 's utility when the tax rate of the next period is $\tau^{\prime}$ and the economy reverts to $\Psi \tilde{v}_{i}(\cdot, \cdot, \cdot ; \Psi)$. I am now ready to determine the policy rule, the $\Psi$ that arises in equilibrium. 
The highest utility achievable for a household of type $i$ is the tax rate that solves

$$
\psi_{i}\left(A, \tau, A_{i} ; \Psi\right)=\operatorname{argmax}_{\tau^{\prime}} \tilde{v}_{i}\left(A, \tau, \tau^{\prime}, A_{i} ; \Psi\right) .
$$

This function returns the most preferred value for the next period's tax rate of agent $i$, given that at all later dates, the tax policy is given by the function $\Psi$.

Now I need a procedure to go from individual most preferred policies to society's policy choice. Such a procedure in this example is majority rule. In this case, if preferences are singlepeaked in $\tau^{\prime}$ for all $i$ and there is only one dimension of heterogeneity (either wealth $a$ or efficiency $\epsilon$ ), a median voter theorem applies. This means that once I label groups so that group 1 is the poorest and group $I$ the richest, the median household will be in the group that has the property that the accumulated share of the population includes the $50 \%$ mark. I refer to the type that includes the median household with an $m$.

The fixed-point condition determining $\Psi$ is thus

$$
\Psi(A, \tau)=\psi_{m}\left(A, \tau, A_{m}: \Psi\right)
$$

for all $A, \tau$.

\section{Computation}

The procedure involves linear quadratic approximations that solve for recursive equilibria for given policies ( $\Psi$ functions). The problem of the median household is then solved given these equilibrium functions, again by using linear-quadratic approximations. If the choice of the median voter coincides with the original $\Psi$ function, an equilibrium is found; if not, I update and continue until convergence occurs.

To do this, note that if all households have the same efficiency, then the pair $\{A, \tau\}=$ $\{K, \cdots, 0\}$ of equal distribution and zero taxes where $K$ satisfies $f^{\prime}(K / L)(1-\tau)=\beta^{-1}$ is a steady state.

\section{Algorithms for Finding Steady States}

I am searching for an $(I-1)$-dimensional subspace of steady states. This means that for every relative distribution of wealth, I want to find its associated steady state. I fix such a relative 
distribution. Then I search for the tax rate associated to that relative wealth distribution. The procedure for computing such a tax rate can be described as follows.

1. Guess on $\tau_{0}$ as a value for the tax rate, and compute the implied steady-state values of the other variables. This involves computing a value for aggregate capital with the property that the after-tax rate of return is the inverse of the discount rate.

2. Let $R_{i}^{0}\left(A, \tau, \tau^{\prime}, a, a^{\prime}\right)$ be a quadratic function that approximates the utility function in a neighborhood of the point where the distribution of wealth has the relative shares for for all types that I fixed, where the total wealth is the one determined in the previous step and where both today's and tomorrow's taxes are set at $\tau_{0}$. Note that the budget constraint has been used here to substitute out consumption.

3. Fix an initial affine tax policy $\Psi^{0}$.

4. Given $\Psi_{0}$, use standard methods to solve for the equilibrium elements $h_{i}^{0}, H^{0}, g_{i}^{0}$, and $G^{0}$ as linear functions and $v_{i}^{0}$ as a quadratic function.

5. Solve for the one-period deviation equilibrium elements. Note that this is a simple static problem, since I already have obtained functions $v_{i}^{0}$. The key difference is that in this case, I do not use $\Psi^{0}$ as an update for the next period's tax rate. Instead, I leave the dependence on $\tau^{\prime}$ explicit. The application of a representative-type assumption on $\tilde{h}_{i}^{0}$, the summing up of the $\tilde{g}_{i}^{0}$ 's, and the matrix inversion then deliver the equilibrium elements $\tilde{H}^{0}$ and $\tilde{G}^{0}$.

6. Substitute the decision rules and the obtained equilibrium functions into the maximand in (56) to obtain the function $\tilde{v}_{i}^{0}$.

7. Maximize $\tilde{v}_{i}^{0}$ with respect to $\tau^{\prime}$ to obtain a function $\psi_{i}^{0}$ of the distribution of wealth and the wealth of the agent. Check for the concavity of the function $\tilde{v}_{i}^{0}$ with respect to $\tau^{\prime}$, ensuring that the first-order conditions deliver a maximum.

8. Use the representative-type condition on the median household to obtain the function $\Psi^{1}$ by letting $\Psi^{1}(A, \tau) \equiv \psi_{m}^{0}\left(A, \tau, A_{m}\right)$.

9. Compare $\Psi^{1}$ with $\Psi^{0}$. If these functions are close enough, continue to step 10 . If not, redefine $\Psi^{0}$ to be a linear combination of its old value and $\Psi^{1}$, and go back to step 4 . This updating procedure has been used before, and it is necessary in our case for avoiding "overshooting" problems. I have found that quite a large step (about 0.9 in the direction of $\Psi^{1}$ ) works well. 
10. Verify that the policy function $\Psi$ reproduces the conjectured tax rate. In other words, the following condition has to be verified:

$$
\tau^{0}=\tau^{1} \equiv \Psi\left(A, \tau^{0}\right)
$$

If it is not, go back to step 1 and update the guess for $\tau$. Update using $\tau_{0}=\left(\tau_{0}+\tau_{1}\right) / 2$.

In these experiments, I use two procedures to characterize the set of steady states. One consists of steps 1 through 9 above. The other procedure, which is much simpler and less timeconsuming, is already described in the description of the mechanics of the model. It is based on the knowledge that a zero tax and an equal distribution constitute a steady state. By using the law of motion for the economy approximated around this point, I can compute the set of steady states by simply finding the set of values for $A_{1}$ and $A_{2}$ that are reproduced by this law of motion. Clearly, this procedure is strictly valid only locally, and it is likely to give lower accuracy further away from the point of perfect equality. Finally, note that this procedure can also be applied to extend locally the set of steady states around any steady-state point found with the first procedure.

\section{Algorithms for Computing Transitional Dynamics}

As for computing steady states, two alternative procedures to compute transitional dynamics can be applied. One uses the linear law of motion for the zero-tax steady state to generate a path for wealth and taxes after a small initial perturbation in asset holdings. The other essentially follows steps 1-8 above; that is, it also involves a separate linearization around each new point the economy passes through. The slight complication needed is an additional round of iterations within step 2: it is necessary to make sure that, at each point on the dynamic path, the point $\left(A, A^{\prime}\right)$ around which the linearization is made coincides with the equilibrium outcome. Note that the equilibrium elements take on different forms for each point on the path, and the accuracy of this procedure is, hence, better the more slowly, or the more linearly, the exact equilibrium evolves. 


\section{References}

Auerbach, A. J. and Kotlikoff, L. J. 1987. Dynamic Fiscal Policy. Cambridge University Press, New York.

Auerbach, A. J., Kotlikoff, L. J., Hagemann, R. P., and Nicoletti, G. 1989. The economic dynamics of an ageing population: the case of four oecd countries. OECD Economic Studies, 12, 97-130.

Castañeda, A., Díaz-Giménez, J., and Ríos-Rull, J.-V. 1997. Unemployment spells, cyclically moving factor shares and income distribution dynamics. Unpublished Manuscript, Federal Reserve Bank of Minneapolis.

Cubeddu, L. M. and Ríos-Rull, J.-V. 1996. Marital risk and capital accumulation. Unpublished Manuscript, University of Pennsylvania.

Díaz-Giménez, J. 1996. Uninsured idiosyncratic risk, liquidity constraints and aggregate fluctuations. Working Paper, Universidad Carlos III de Madrid.

Díaz-Giménez, J., Prescott, E. C., Fitzgerald, T., and Alvarez, F. 1992. Banking in computable general equilibrium economies. Journal of Economic Dynamics and Control, 16, 533-559.

Hansen, G. D. and Prescott, E. C. 1995. Recursive methods for computing equilibria of business cycle models. In Cooley, T. F. (Ed.), Frontiers of Business Cycle Research, chap. 2. Princeton University Press, Princeton.

Huang, H., Imrohoroğlu, S., and Sargent, T. J. 1997. Two computations to fund social security. Forthcoming, Macroeconomic Dynamics.

Huggett, M. 1993. The risk free rate in heterogeneous-agents, incomplete-insurance economies. Journal of Economic Dynamics and Control, 17(5/6), 953-970.

Imrohoroğlu, A., Imrohoroğlu, S., and Joines, D. 1994. Effect of tax-favored retirement accounts on capital accumulation and welfare. Unpublished Manuscript, University of Southern California.

Judd, K. L. 1991. Numerical methods in economics. Unpublished Manuscript, Hoover Institution.

Krusell, P., Quadrini, V., and Ríos-Rull, J.-V. 1996. Are consumption taxes really better than income taxes?. Journal of Monetary Economics, 37(3), 475-504.

Krusell, P., Quadrini, V., and Ríos-Rull, J.-V. 1997. Politico-economic equilibrium and economic growth. Journal of Economic Dynamics and Control, 21(1), 243-272.

Krusell, P. and Ríos-Rull, J.-V. 1994. What constitutions promote capital accumulation? a political economy approach. Unpublished Manuscript, University of Pennsylvania.

Krusell, P. and Smith, A. 1995a. Income and wealth heterogeneity in the macroeconomy. Working Paper 399, Rochester Center for Economic Research.

Krusell, P. and Smith, A. 1995b. Income and wealth heterogeneity, portfolio choice, and equilibrium asset returns. Unpublished Manuscript, University of Rochester.

Ríos-Rull, J.-V. 1992. Business cycle behavior of life-cycle economies with incomplete markets. Cuadernos Economicos de ICE, 51(2), 173-96. 
Ríos-Rull, J.-V. 1994a. On the quantitative importance of market completeness. Journal of Monetary Economics, 34, 463-96.

Ríos-Rull, J.-V. 1994b. Population changes and capital accumulation: the aging of the baby boom. Unpublished Manuscript, University of Pennsylvania.

Ríos-Rull, J.-V. 1995. Models with heterogenous agents. In Cooley, T. F. (Ed.), Frontiers of Business Cycle Research, chap. 4. Princeton University Press, Princeton.

Ríos-Rull, J.-V. 1996. Life cycle economies and aggregate fluctuations. Review of Economic Studies, 63, 465-490.

Stokey, N. L. and Lucas, R. E. with Prescott, E. C. 1989. Recursive Methods in Economic Dynamics. Harvard University Press.

Storesletten, K. 1994. Sustaining fiscal policy through immigration. Unpublished Manuscript, Carnegie Mellon University.

Trick, M. A. and Zin, S. E. 1993. A linear programming approach to solving stochastic dynamic programs. Unpublished Manuscript, Carnegie Mellon University.

Uhlig, H. 1996. A law of large numbers for large economies. Economic Theory, 8(1), 41-50. 